\title{
Foundation Document for the European Family Support Network (EFSN)
}

\section{Overview}

Families provide the primary site for caring for children whom they raise not for their benefit alone but for the betterment of civic society. Not enough is known or being done to support families in carrying out the complex task of rearing children and young people. In an increasingly challenging and changing environment there is a need for the international practice, policy and academic community to respond.

The EFSN is a network that is a champion family support research, theory, policy and practice development across Europe. We share a value-based commitment to supporting families across Europe. We believe that there is an evidence base for high quality family support practice and that all relevant policies and practices should be evaluated from the perspective of their contributions to family support. We believe that State and European policies have a crucial role to play in supporting families to raise their children.

\section{Objectives}

The EFSN is an inclusive organisation, which aims to promote the following objectives:

- Undertake, facilitate and support research and other initiatives, which champions family support policy, practice and service delivery.

- Contribute to theoretical and conceptual development in relation to supporting families

- Provide a focus on children, young people, parent/carers and family networks as active participants in the Network and in the family support process more generally

- To take a multi-method and multi-disciplinary approach to our research and related activities

- Influence State governments, NGOs and professionals to recognise the importance of policy to support families more effectively 


\section{Working at three levels}

Policies and practices should recognize and acknowledge that all families need support in order to undertake their role.

The EFSN adopts a holistic approach to service development at the following three levels

\section{Level 1. Universal}

Provision to support all families in raising children and young people

\section{Level 2. Secondary}

Services for those facing challenges shared by many families, which can be addressed through accessible, non-stigmatising and flexible service provision

\section{Level 3. Tertiary}

Services for those requiring support in addressing complex challenges

This is achieved in the main through familial and community based informal sources of support, as well as semi-formal and formal professional services particularly at the tertiary level.

\section{Working principles}

The work of the ESFN is underpinned by the following set of principles:

- We are inclusive of all who wish to play an active and positive role in supporting families in all their forms

- We wish to promote participation by children, young people, parents/carers and the extended family network in all our work

- We are evidence-based - drawing on a wide-range of research evidence and methods.

- We support the effective use of cash transfers to families working alongside service delivery in a fit for purpose and integrated manner

- We believe that family support has an ethical base in promoting human dignity social inclusion, human rights and the rights of children and young people.

- We support open access to all EFSN written and web-based resources

- We aim to be a dynamic, responsive, inclusive and sustainable network 


\section{Theoretical and conceptual underpinnings}

A shared and common definition of family support is an emerging field that requires further conceptual and theoretical development. The EFSN aims to contribute to this knowledge creation by developing a working definition of family support.

Whilst we are not dogmatic about the use of particular concepts, we promote open discussion and the exploration and development of all theories and concepts, which help, illuminate family support.

The Network takes an inclusive stance on social, psychological and psychosocial theories embedded in an ecological approach that helps to illuminate our projects. Social support, social inclusion, social ecology and resilience lay at the heart of our approach. Our approach is consistent with reflective, relational and restorative professional practices.

The Network supports and utilise a mixed method, multi-disciplinary approach to research and related activities, thus building a solid and diverse evidence base for family support.

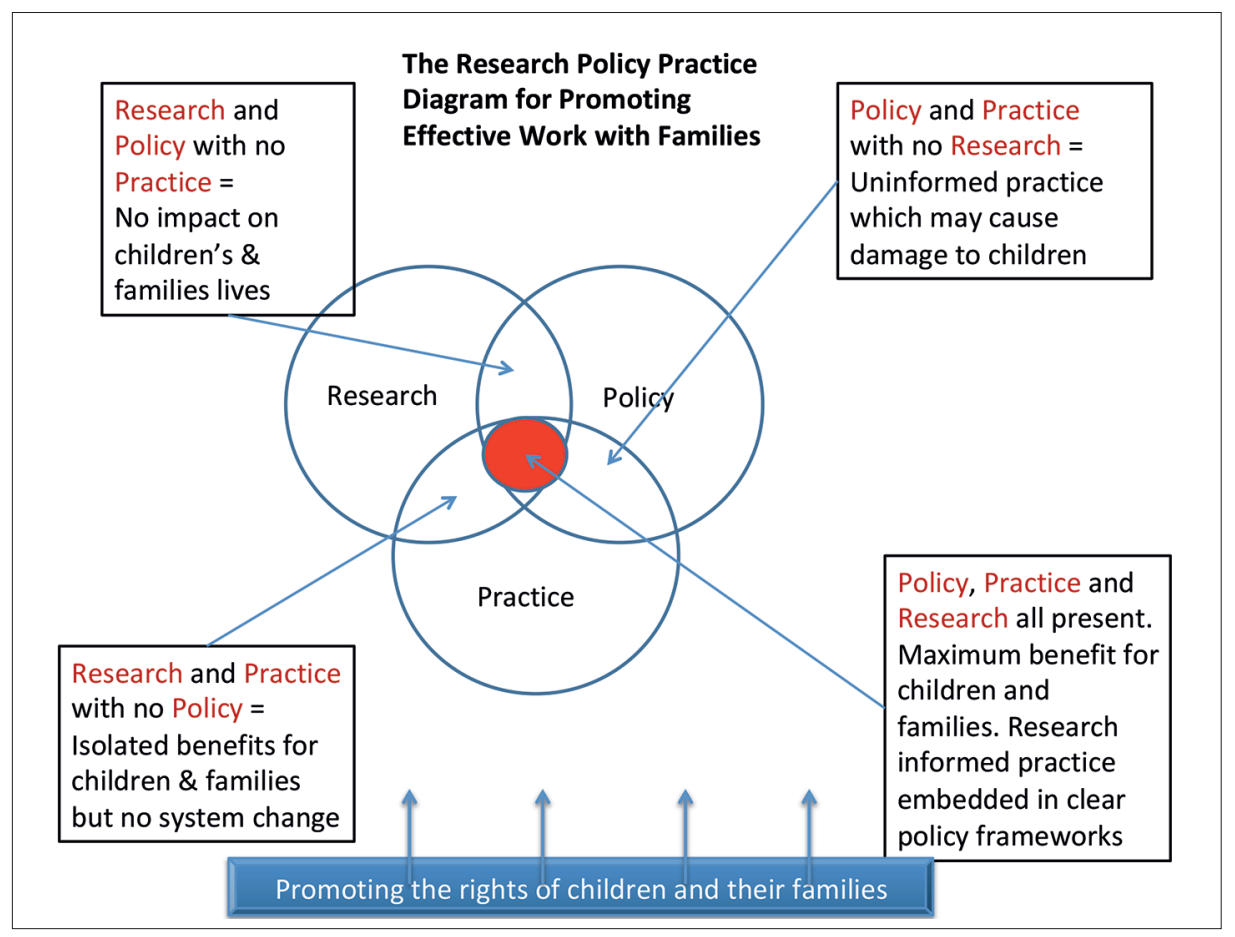

